\title{
BKM120 sensitizes C6 glioma cells to temozolomide via suppression of the PI3K/Akt/NF-кB/MGMT signaling pathway
}

\author{
MAO LI, RUO FEI LIANG, XIANG WANG, QING MAO and YAN HUI LIU \\ Department of Neurosurgery, West China Hospital, Sichuan University, Chengdu, Sichuan 610000, P.R. China
}

Received December 7, 2015; Accepted May 19, 2017

DOI: $10.3892 / 01.2017 .7034$

\begin{abstract}
Glioblastoma is the most common type of malignant intracranial tumor in adults. Temozolomide (TMZ), as the first-line chemotherapy agent used in patients with glioblastoma, has demonstrated different effects in patients due to the expression of O6-methylguanine-DNA methyltransferase (MGMT) which is able to repair the DNA lesions induced by TMZ. The phosphatidylinositol 3-kinase (PI3K)/Akt signaling pathway is over-activated in glioblastoma and has been revealed to be potentially implicated in resistance to TMZ. BKM120, a selective pan class I PI3K inhibitor, has been reported to facilitate apoptosis and reverse drug resistance in advanced solid tumors. The present study aims to investigate whether BKM120 is able to sensitize glioma cells to TMZ. C6 glioma cells were treated with BKM120 and/or TMZ for 12, 24 and $48 \mathrm{~h}$, respectively. Cell Counting Kit- 8 assays were performed to determine cell viability. The level of apoptosis was evaluated by Hoechst 33342 and TUNEL staining, and the levels of cleaved caspase-3 and Bcl-2-like protein 4 (Bax) expression was measured. Furthermore, the present study investigated the possible mechanism underlying BKM120 reverse chemoresistance to TMZ. The downstream targets of PI3K, including phosphorylated (p)-Akt, nuclear factor (NF)-B p65, were analyzed by western blotting. The MGMT transcription levels in monotherapy and combination therapy were demonstrated by reverse transcription-quantitative polymerase chain reaction (RT-qPCR). The present study revealed that monotherapy treatments with either drug required a high concentration found reduction in cell viability. However, a low concentration of BKM120 inhibited the PI3K/Akt/NF- $\mathrm{B}$ signaling pathway activity in glioma cells and significantly increased TMZ cytotoxicity. The
\end{abstract}

Correspondence to: Professor Yan Hui Liu, Department of Neurosurgery, West China Hospital, Sichuan University, 37 Guoxue Xiang, Chengdu, Sichuan 610000, P.R. China

E-mail: yhliu2001@gmail.com

Key words: glioma, temozolomide, BKM120, chemotherapy, apoptosis, O6-methylguanine-DNA methyltransferase, nuclear factor- $\kappa \mathrm{B}$ coefficient of drug interaction was $\sim 0.7$. Results from the TUNEL assay, Hoechst 33342 staining and evaluation of the levels of cleaved caspase- 3 and Bax expression also confirmed the finding that the combination treatment induced a higher level of apoptosis compared with the TMZ monotherapy. RT-qPCR demonstrated that the combination strategy reversed the TMZ-induced MGMT over-transcription. The reduction of $\mathrm{NF}-\kappa \mathrm{B}$ p 65 in combination treatment supported the hypothesis that BKM120 may mediate MGMT transcription via inhibition of $\mathrm{NF}-\kappa \mathrm{B}$ p65. In conclusion, BKM120 and TMZ demonstrated strong synergistic cytotoxicity in C6 glioma cells. The BKM120-induced NF- $\kappa$ B p65 inhibition may be involved in the mediation of MGMT transcription to reverse TMZ-resistance in C6 glioma cells.

\section{Introduction}

Glioblastoma is one of the most common types of malignant tumors and exhibits a poor prognosis (1). Due to the high invasiveness and heterogeneity, glioma has been demonstrated to exhibit resistance to traditional treatment, including surgery, irradiation and chemotherapy (1). The average survival time for patients with glioblastoma has marginally changed over the previous years and remains at $\sim 8-12$ months (2). Therefore, there is an urgent requirement to develop novel therapy strategies in order to treat this malignant neoplasm.

Temozolomide (TMZ) is an orally administered alkylating agent with promising antitumor activity and ability to cross the brain-blood barrier (2). Randomized clinical trials have been performed to demonstrate that TMZ-based standard chemotherapy may significantly improve survival time and quality of life for patients with glioblastoma (3). The mechanism of action of TMZ is attributed to cell apoptosis due to induction of DNA O6-methyl guanine. However, treatment with TMZ alone is insensitive to specific patients who highly express the O6-methylguanine-DNA methyltransferase (MGMT). MGMT acts as a transferase and removes the cytotoxic O6-alkylguanine DNA adducts induced by TMZ (4). Previous studies have revealed that phosphatidylinositol 3-kinase (PI3K)/AKT/mechanistic target of rapamycin signaling pathway over-activation is a common event in glioma and its downstream targets, including p53, nuclear factor $(\mathrm{NF})-\kappa \mathrm{B}$. p53 and NF- $\mathrm{B}$ contribute to chemotherapy resistance by mediating the transcription of a number of genes, including MGMT (5). 
NVP-BKM120, an oral highly selective pan-class I PI3K inhibitor, has been used in previous studies to treat various solid types of cancer and demonstrated an ability to induce cancer cell apoptosis and reverse chemoresistance. In phase I clinical trials, NVP-BKM120 was demonstrated to suppress tumor growth and proliferation at tolerated doses in various types of solid cancer (6).

In the present study, the authors hypothesized that targeting the PI3K/AKT signaling pathway by BKM120 in combination with TMZ may be a promising strategy for the treatment of glioma. Furthermore, the present study investigated the possible mechanism underlying the PI3K/AKT signaling pathway and drug resistance.

\section{Materials and methods}

Tumor cell line. To investigate the TMZ-resistance mechanism of glioma cells, the present study used the C6 glioma cell line, a MGMT-positive cell line (Type Culture Collection of the Chinese Academy of Sciences, Shanghai, China). In the present study, glioma cells were cultured in Dulbecco's modified Eagle's medium (Invitrogen; Thermo Fisher Scientific, Inc., Waltham, MA, USA) supplemented with $10 \%$ fetal bovine serum (Gibco; Thermo Fisher Scientific, Inc.) at $37^{\circ} \mathrm{C}$ in an atmosphere containing $5 \% \mathrm{CO}_{2}$ for 24 or $48 \mathrm{~h}$.

Reagents. TMZ was supplied by Tasly Pharmaceutical Co., Ltd. (Tianjin, China). The PI3K inhibitor, BKM120, was purchased from Sigma-Aldrich (Merck KGaA, Darmstadt, Germany). Stock solutions of TMZ and BKM120 (TMZ, $120 \mathrm{mM}$; BKM120, 3 mM) were made by dissolving in dimethyl sulfoxide (DMSO; Sigma-Aldrich; Merck KGaA). For TMZ treatment solutions, the DMSO concentrations were limited to $<1 \%(\mathrm{v} / \mathrm{v})$, and the vehicle controls were added at the same concentration as DMSO. The terminal deoxynucleotidyl transferase dUTP nick end labeling (TUNEL) assay cell death kit was purchased from Roche Diagnostics (Indianapolis, IN, USA). The Cell Counting Kit-8 (CCK-8) and Hoechst 33342 were supplied by Beyotime Institute of Biotechnology (Haimen, China). All primary antibodies were purchased from Cell Signaling Technology, Inc. (Danvers, MA, USA), including rabbit anti-rat antibodies against PI3K (cat. no. 4257, 1:1,000 dilution), phosphorylated (p)-Akt $\left(\operatorname{Ser}^{473}\right)$ (cat. no. 4060, 1:1,000 dilution), NF-кB p65 (cat. no. 3033, 1:1,000 dilution), pS6 (cat. no. 2215S, 1:1,000 dilution), cleaved caspase-3 (cat. no. 9664, 1:1,000 dilution), Bax (cat. no. 14796, 1:1,000 dilution) and GAPDH (cat. no. 5174, 1:1,000 dilution,), and goat anti-rabbit horseradish peroxidase (HRP)-conjugated immunoglobulin G (IgG) secondary antibody (cat. no. 31460, 1:1,000 dilution; Pierce; Thermo Fisher Scientific, Inc.).

Cell viability assay. Cell viability and coefficient of drug interactions (CDIs) were determined by CCK- 8 assay. The cells were seeded with a density of $5 \times 10^{3}$ cells/well in 96-well plates and incubated, at $37^{\circ} \mathrm{C}$, in complete medium (DMEM with $20 \% \mathrm{FBS}$ ) with serial dilutions of TMZ and/or BKM120 for $24 \mathrm{~h}$. Tumor cells were divided into four groups (TMZ alone, BKM120 alone, TMZ and BKM120 combined, vehicle control). In the TMZ treatment group, TMZ was added to the culture medium at final concentrations of 300, 600, 900 and 1,200 $\mu \mathrm{M}$. In the BKM120 treatment groups, BKM120 was added to culture medium at final concentrations of 300,600 , 1,500 and $3,000 \mathrm{nM}$. In the combination treatment group, BKM120 (300 nM) was added to culture mediums in combination with various concentrations of TMZ $(300,600,900$ and $1,200 \mu \mathrm{M})$. Following incubation at $37^{\circ} \mathrm{C}$ for 12,24 and $48 \mathrm{~h}$, CCK-8 solution was added into each well with a final concentration of $20 \mu \mathrm{l} /$ well. Following incubation for $4 \mathrm{~h}$, absorbance was determined at $490 \mathrm{~nm}$ using a microplate reader. Cell viabilities and CDIs were evaluated using the data from the independent CCK- 8 assay results. The $\mathrm{IC}_{50}$ value was defined as the mean drug concentration required for inhibiting $50 \%$ of cell viability compared with the vehicle controls.

Hoechst 33342 staining. Briefly, C6 glioma cells were plated in 6 -well plates with a density of $1 \times 10^{5}$ cells/well and treated with TMZ (300 $\mu \mathrm{M})$ and/or BKM120 (300 nM) at $37^{\circ} \mathrm{C}$ for $24 \mathrm{~h}$. The cells were washed in PBS three times and incubated in Hoechst 33342 solution $(10 \mu \mathrm{g} / \mathrm{ml})$ for $30 \mathrm{~min}$ at $4^{\circ} \mathrm{C}$. Finally, fluorescence microscopy (magnification, x40) was preformed to observe the nuclear changes of C6 glioma cells. For each treatment group, $\geq 1,000$ cells were analyzed in triplicate.

TUNEL staining. Following treatment with drugs (TMZ, $300 \mu \mathrm{M}$; BKM120, $300 \mathrm{nM}$ ) for $24 \mathrm{~h}$ and washing with PBS three times, the cells were incubated with stationary liquid (4\% paraformaldehyde) for $30 \mathrm{~min}$, and subsequently treated with $3 \%$ hydrogen peroxide for 10 min to block endogenous peroxides at room temperature. Following washing with PBS, $0.1 \%$ Triton was added to perforate the cells for $10 \mathrm{~min}$ at room temperature. Subsequently, the slides were incubated with terminal deoxynucleotidyl transferase enzyme for $1 \mathrm{~h}$ at room temperature. Gold antifade mountant with DAPI (P36935; Invitrogen; Thermo Fisher Scientific, Inc.) was used to mount the slides, according to the manufacturer's instructions. Images were captured using a microscope camera system (BX63; Olympus, Tokyo, Japan) (magnification, x40).

Western blot analysis. Following drug treating with TMZ $(300 \mu \mathrm{M})$ and/or BKM120 (300 nM) for $24 \mathrm{~h}$ at $37^{\circ} \mathrm{C}$, proteins were extracted using a cell lysis buffer (PBS, 1\% Triton, 1\% SDS, protease inhibitor cocktail III (539134; Merck KGaA), phosphatase inhibitor cocktail III (k276-1EA; BioVision, Inc., Milpitas, CA, USA). The proteins were centrifuged for $10 \mathrm{~min}$ at $10,000 \mathrm{x} \mathrm{g}$ at $4^{\circ} \mathrm{C}$ and protein was determined using the bicinchoninic assay method. The proteins $(10 \mu \mathrm{g} /$ lane) were separated using $12 \%$ SDS-PAGE (15\% gel for cleaved caspase-3) and subsequently transferred to nitrocellulose membranes. Nitrocellulose membranes were blocked with $5 \%$ milk for $2 \mathrm{~h}$ at $37^{\circ} \mathrm{C}$ and subsequently incubated with primary antibodies against PI3K, pAkt $\left(\mathrm{Ser}^{473}\right)$, pS6, cleaved caspase-3, Bax and GAPDH at $4^{\circ} \mathrm{C}$ overnight. The membranes were subsequently washed with Tris-buffered saline supplemented with $1 \%$ Tween-20 three times and incubated with goat anti-rabbit immunoglobulin $\mathrm{G}$ at $37^{\circ} \mathrm{C}$ for $1 \mathrm{~h}$. In addition, the enhanced chemiluminescence western blotting detection system (Medicare X-Ray-Processor 102; Kodak, Rochester, NY, USA) was used to visualize antibody complexes. Finally, protein densities were measured using 

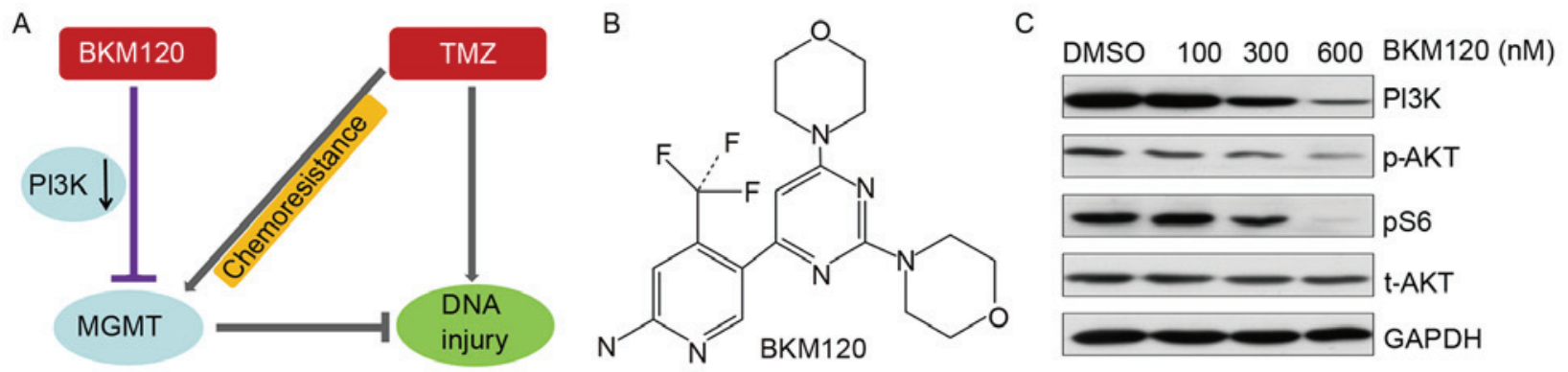

Figure 1. (A) Hypothesized mechanism of action of the BKM120 and TMZ combination treatment. (B) Chemical structure of BKM120. (C) C6 glioma cells were treated with increased doses of BKM120 (100,300 and $600 \mathrm{nM})$ and DMSO for $24 \mathrm{~h}$, respectively. The expression of PI3K, p-Akt and pS6 was reduced in BKM120 treated C6 cells. TMZ, temozolomide; DMSO, dimethyl sulfoxide; MGMT, O6-methylguanine-DNA methyltransferase; PI3K, phosphatidylinositol 3-kinase; p, phosphorylated; Akt, protein kinase B; t, total protein.

ImageJ software (version 2.0; National Institutes of Health, Bethesda, MD, USA).

Reverse transcription-quantitative polymerase chain reaction $(R T-q P C R)$. For RT-qPCR, C6 glioma cells were collected following treatment with TMZ $(300 \mu \mathrm{M})$ and/or BKM120 (300 $\mathrm{nM}$ ) for $24 \mathrm{~h}$, and subsequently $\geq 1 \mathrm{mg}$ total RNA was extracted from individual samples using TRIzol ${ }^{\circledR}$ reagent (Invitrogen; Thermo Fisher Scientific, Inc.), according to the manufacturer's instructions. All RNAs were reverse transcribed into cDNA using the Revert Aid First Strand cDNA Synthesis kit (Thermo Fisher Scientific, Inc.) for RT-PCR. cDNA was added to a total volume of $20 \mu \mathrm{l}$. qPCR was performed using the SYBR Premix Ex Taq ${ }^{\mathrm{TM}}$ II kit (Takara Biotechnology, Co., Ltd., Dalian, China) for MGMT and $\beta$-actin. The $2^{-\Delta \Delta C q}$ method (7) was used to determine the relative mRNA expression levels of MGMT, with $\beta$-actin mRNA as an endogenous control. All experiments were repeated three times.

The temperature protocol for qPCR was as follows: i) $50^{\circ} \mathrm{C}$ for $2 \mathrm{~min}, 1 \mathrm{cycle}$; ii) $95^{\circ} \mathrm{C}$ for $10 \mathrm{~min}, 1 \mathrm{cycle}$; iii) $95^{\circ} \mathrm{C}$ for $15 \mathrm{sec}, 60^{\circ} \mathrm{C}$ for $30 \mathrm{sec}$ and $72^{\circ} \mathrm{C}$ for $30 \mathrm{sec}, 40$ cycles; and iv) $72^{\circ} \mathrm{C}$ for $10 \mathrm{~min}, 1$ cycle. The temperature protocol for reverse transcription was as follows: i) $25^{\circ} \mathrm{C}$ for $5 \mathrm{~min}$; ii) $42^{\circ} \mathrm{C}$ for $30 \mathrm{~min}$; and iii) $85^{\circ} \mathrm{C}$ for $5 \mathrm{~min}$. Primer sequences used were as follows: MGMT mRNA forward, 5'-AGGAGC GATGAGGAGCAATC-3' and reverse, 5'-CAGACTAAC CCTCGACCGAG-3'; $\beta$-actin mRNA forward, 5'-GAGACC TTCAACACCCCAGC-3' and reverse, 5'-CCCTTTAGCACG CACTGTA-3'.

Statistical analysis. Each analysis was repeated for $\geq 3$ independent experiments. Statistical analysis of results was performed using SPSS software version 21 (IBM Corp., Armonk, NY, USA). All data were tested for significance by unpaired Student's t-test or one-way analysis of variance (ANOVA). Tukey's test and Dunnett's correction were performed following ANOVA. $\mathrm{P}<0.05$ was considered to indicate a statistically significant difference. All data are presented as the mean \pm standard deviation.

\section{Results}

Synergistic cytotoxicity from combination of BKM120 and TMZ in C6 glioma cells. According to the hypothesis of the present study (Fig. 1A and B), the present study verified the PI3K-induced inhibition of BKM120 in C6 glioma cells (Fig. 1C) and subsequently performed CCK-8 analysis to detect the synergistic effect of BKM120 and TMZ. C6 glioma cells were treated alone with increasing doses of TMZ or BKM120 for 12, 24 and $48 \mathrm{~h}$. There were decreased cell viabilities at 24 and $48 \mathrm{~h}$, compared with that observed at $12 \mathrm{~h}$ (Fig. 2A). The $\mathrm{IC}_{50}$ of TMZ (24 h, 1,137.67 $\pm 271.53 \mu \mathrm{M} ; 48 \mathrm{~h}$, $969.09 \pm 269.61 \mu \mathrm{M})$ and BKM120 $(24 \mathrm{~h}, 4.78 \pm 1.63 \mu \mathrm{M} ; 48 \mathrm{~h}$, $2.20 \pm 0.64 \mu \mathrm{M}$ ) achieved a high value, which suggested that $\mathrm{C} 6$ glioma cells are insensitive to TMZ or BKM120 monotherapy.

However, when cells were treated with the combination treatment, which comprised a low concentration of BKM120 (300 $\mathrm{nM})$ and various concentrations of TMZ (300, 600, 900 and $1,200 \mu \mathrm{M}$ ) for 24 and $48 \mathrm{~h}$, the $\mathrm{IC}_{50}$ of TMZ was significantly decreased $(24 \mathrm{~h}, 1,137.67 \pm 271.53 \mu \mathrm{M}$ to $346.67 \pm 134.53 \mu \mathrm{M}$; $48 \mathrm{~h}, 969.09 \pm 269.61$ to $794.75 \pm 314.09 \mu \mathrm{M} ; \mathrm{P}<0.05$, unpaired Student's t-test; Fig. 2B and C). The CDI values were close to 0.7 , which indicated that BKM120 increased the toxicity of TMZ (Table I). Furthermore, the present study confirmed that the inhibition of PI3K was induced by BKM120 in C6 glioma cells, and the expression levels of its downstream targets (p-AKT, pS6) were also decreased (Fig. 1C).

BKM120 increases TMZ-induced apoptosis of C6 glioma cells. Hoechst 33342 staining assay was performed to determine the level of apoptosis in cells following monotherapy or combination therapy. In the combination treatment group, C6 glioma cells exhibited more nuclear apoptotic changes, including nuclear condensation and aggregation (Fig. 3A). The rate of Hoechst 33342-positive cells in the combination treatment group was significantly increased, compared with that of the TMZ group or the BKM120 group (44.06 \pm 7.28 vs. $17.68 \pm 3.04 \%$ and $13.37 \pm 2.23 \%$, respectively; $\mathrm{P}<0.05$, one-way ANOVA; Fig. 3B). In addition, TUNEL staining was performed to quantify the fraction of cells that were apoptotic. The analysis indicated that the immunofluorescence intensity of the combination treatment group was significantly $(\mathrm{P}<0.05$, ANOVA) higher compared with the TMZ group or the BKM120 group (Fig. 4A and B). The present study also determined the level of expression of apoptosis-associated proteins (cleaved caspase- 3 and Bax) by western blotting. The results confirmed that the combination strategy induced increased expression of cleaved caspase- 3 and Bax expression compared 

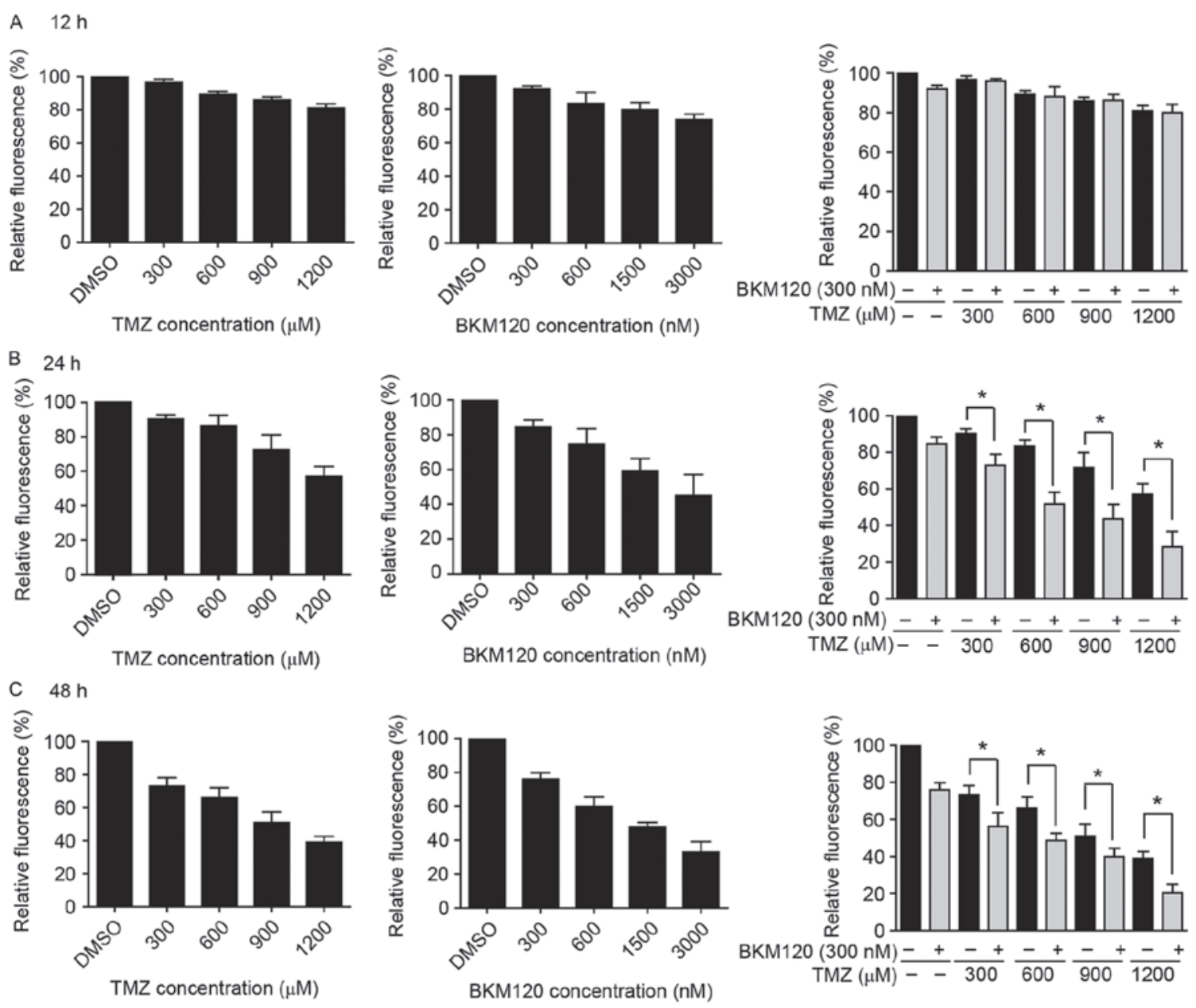

Figure 2. (A) Relative fluorescence of C6 glioma cells treated with TMZ and/or BKM120 for 12 h. (B) Relative fluorescence of C6 glioma cells treated with TMZ and/or BKM120 for $24 \mathrm{~h}$. (C) Relative fluorescence of C6 glioma cells treated with TMZ and/or BKM120 for 48 h. Combination treatment with a low concentration of BKM120 (300 nM) and various concentrations of TMZ (300, 600, 900 and 1,200 $\mu \mathrm{M})$ induced a significant reduction compared with TMZ monotherapy in 24 and $48 \mathrm{~h}\left({ }^{*} \mathrm{P}<0.05\right)$. TMZ, temozolomide.

Table I. Coefficient of drug interaction of combination treatment with TMZ and BKM120.

\begin{tabular}{lcc}
\hline TMZ, $\mu \mathrm{M}$ & BKM120, nM & CDI \\
\hline 300 & 300 & $0.81 \pm 0.05$ \\
600 & 300 & $0.73 \pm 0.08$ \\
900 & 300 & $0.65 \pm 0.06$ \\
1,200 & 300 & $0.81 \pm 0.03$ \\
\hline
\end{tabular}

TMZ, temozolomide; CDI, coefficient of drug interaction.

with TMZ or BKM120 monotherapy (Fig. 3C). These results suggest that BKM120 increased TMZ-induced apoptosis in C6 glioma cells.

BKM120 suppresses TMZ-induced MGMT transcription. RT-qPCR analysis demonstrated that exposure to increasing doses of TMZ for $24 \mathrm{~h}$ significantly increased the level of MGMT transcription. C6 glioma cells exposed to BKM120 alone revealed no significant effects on the level of MGMT transcription (Fig. 5A and B). However, when the cells were treated with a combination of $300 \mathrm{nM}$ BKM120 and $300 \mathrm{nM}$ TMZ, the level of MGMT transcription was significantly downregulated compared with treatment with TMZ monotherapy $(\mathrm{P}=0.03$, one-way ANOVA; Fig. 5C). Taken together, these experimental results demonstrated that BKM120 suppressed TMZ-induced MGMT transcription in C6 glioma cells.

$N F-\kappa B$ p 65 contributes to the therapeutic potential of combination strategy. The mechanism of the observed reversal in TMZ-resistance was further investigated by detecting the levels of $\mathrm{NF}-\kappa \mathrm{B}$ p 65 expression. $\mathrm{NF}-\kappa \mathrm{B}$ p 65 is a downstream of PI3K and an important mediator of MGMT transcription. Using western blotting, it was observed that the expression of $\mathrm{NF}-\kappa \mathrm{B}$ p65 was increased in the TMZ monotherapy group compared with the combination treatment group. It was also indicated that the expression of $\mathrm{NF}-\kappa \mathrm{B}$ p65 was significantly decreased in the TMZ and BKM120 combination treatment group from $139.8 \pm 15.1$ to $54.6 \pm 26.1 \%$ compared with the TMZ monotherapy group ( $\mathrm{P}=0.008$, one-way ANOVA), which was consistent with the changes observed in the level of MGMT transcription (Fig. 5D). This result suggested that $\mathrm{NF}-\kappa \mathrm{B}$ p65 contributed to the therapeutic potential of the combination strategy. 
A

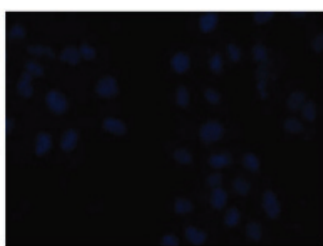

DMSO

B

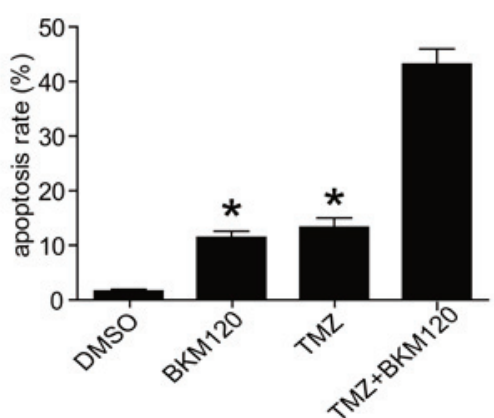

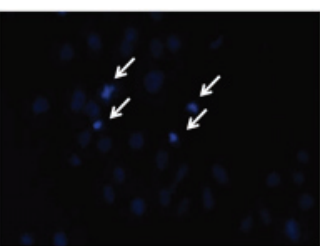

BKM120

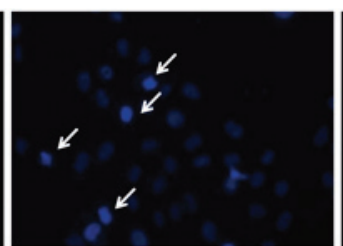

TMZ

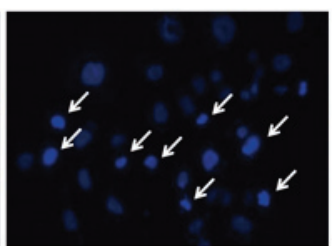

TMZ+BKM120

C

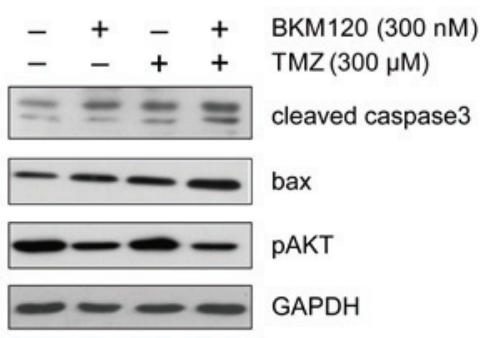

Figure 3. (A) Hoechst 33342 staining assays demonstrated that combination treatment increased morphological changes in the nucleus. The arrows indicate positive cells. (B) The number of Hoechst 33342-positive cells was significantly higher in the combination treatment group compared with the TMZ group or the BKM120 monotherapy group $\left(44.06 \pm 7.28\right.$ vs. $\left.17.68 \pm 3.04,13.37 \pm 2.23 ;{ }^{*} \mathrm{P}<0.05\right)$. (C) Western blotting was used to detect the level of expression of cleaved caspase-3, Bax, p-Akt and GAPDH in cells treated alone with TMZ or BKM120 and with the combination treatment. Bax, Bcl-2-like protein 4; DMSO, dimethyl sulfoxide; TMZ, temozolomide; p, phosphorylated; Akt, protein kinase B.

A

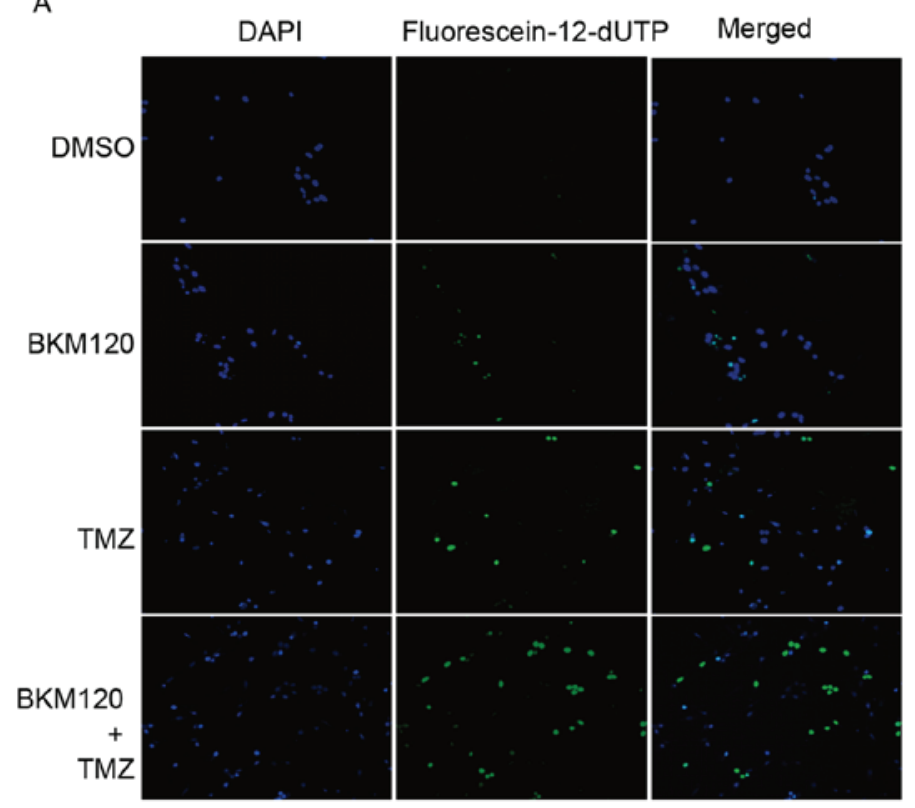

B

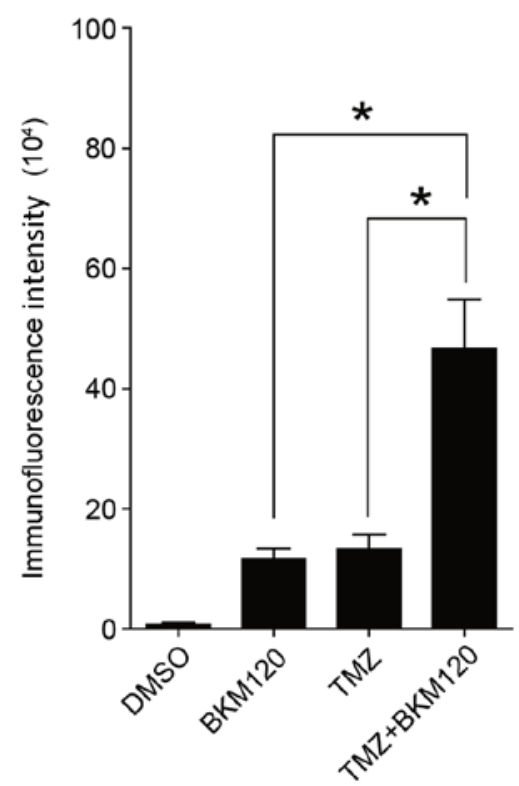

Figure 4. (A) C6 cells were incubated with DMSO, TMZ, BKM120 and TMZ/BKM120 combination treatment for 24 h. TUNEL staining was subsequently performed for detection of apoptotic cells. The nuclei of TUNEL-positive cells were stained green. (B) The immunofluorescence intensity of TUNEL-positive cells in 3 randomly selected samples from each group was analyzed using Image-Pro Plus software. The immunofluorescence intensity of combination treatment group was significantly higher compared with the TMZ and BKM120 monotherapy groups ( $\left.{ }^{*}<0.05\right)$. DMSO, dimethyl sulfoxide; TMZ, temozolomide; TUNEL, terminal deoxynucleotidyl transferase dUTP nick-end labeling.

\section{Discussion}

Glioblastoma is the most rapidly growing and aggressive type of intracranial neoplasm in adults (8). Surgical resection, radiotherapy and TMZ chemotherapy constitute standard therapy for patients with glioblastoma (9). TMZ is able to cross the brain-blood barrier and achieve therapeutic concentration in brain tissues. However, glioblastoma is often resistant to TMZ due to MGMT expression in glioma cells (10). MGMT is highly expressed in solid types of cancer, and its expression removes the TMZ-induced cytotoxic O6-alkylguanine DNA adducts. MGMT promoter methylation status is an important 


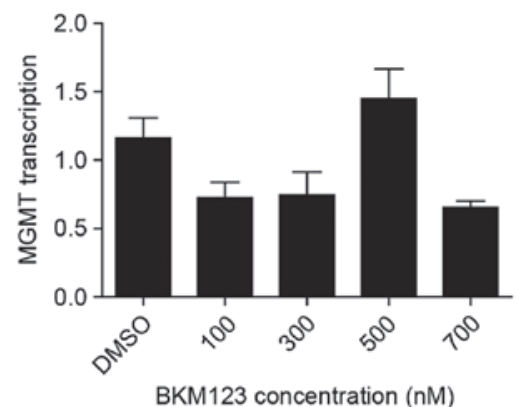

C

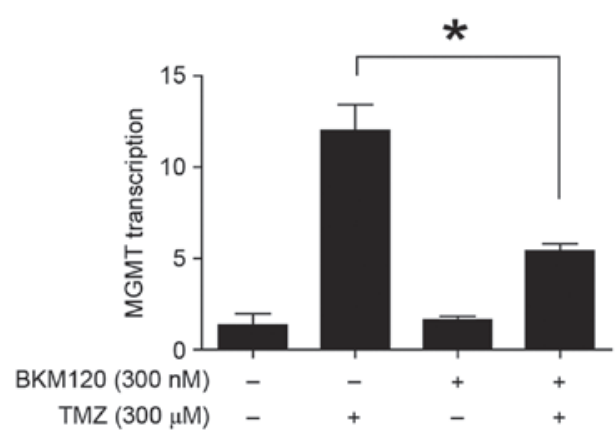

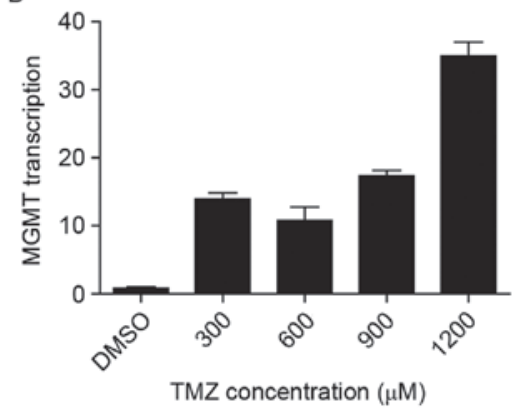

D

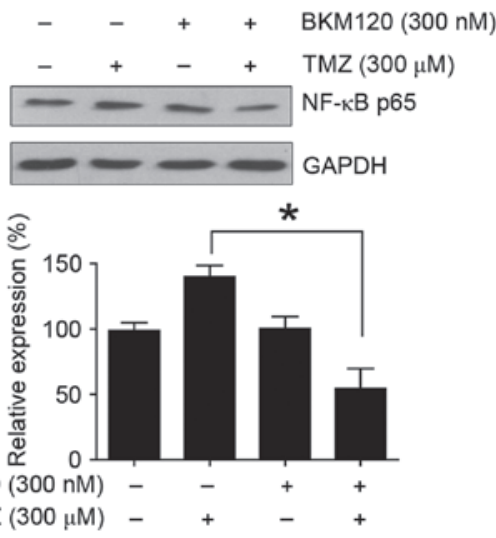

Figure 5. (A) Reverse transcription-quantitative polymerase chain reaction results revealed that the levels of MGMT transcription in C6 glioma cells increased following treatment with TMZ. (B) There was no significant change in the BKM120 monotherapy group. (C) The levels of MGMT transcription in C6 glioma cells treated with a combination of BKM120 and TMZ significantly increased compared with the TMZ monotherapy group (*P=0.03). (D) The combination treatment also induced a marked decrease in NF- $\kappa \mathrm{B}$ p65 expression compared with the TMZ monotherapy group ( $\mathrm{P}<0.05$ ). TMZ, temozolomide; DMSO, dimethyl sulfoxide; MGMT, O6-methylguanine-DNA methyltransferase.

prognosis indicator for patients with glioblastoma (11). Therefore, investigating combination treatments of TMZ with other drugs is required in order to inhibit unexpected DNA repair. A number of MGMT inhibitors, including O6-benzylguanine, streptozotocin and PaTrin-2, have been studied in combination treatment with TMZ to develop a novel chemotherapy strategy to sensitize cancer cells to TMZ (12). However, although these drug combinations are able to effectively inhibit MGMT expression in cancer cells, serious drug reactions and systematic toxicity indicated a disconsolate future (13). There is an urgent requirement to develop well-tolerated drugs with the capacity to reverse TMZ-resistance. Therefore, drugs targeting proteins with the capacity to mediate MGMT expression levels are actively studied.

A previous study demonstrated that over-activation of the PI3K/AKT signaling pathway in glioblastoma was associated with chemotherapy resistance (14). p-Akt is the primary mediator of PI3K-initiated signaling, and a number of its downstream substrates contribute to the resistance of chemotherapy, including Bcl2-associated agonist of cell death, $\mathrm{p} 53, \mathrm{NF}-\kappa \mathrm{B}$ and glycogen synthase kinase-3 $\beta$ (15). NF- $\kappa \mathrm{B}$ is a component, which initiates the inflammatory transcription pathway in various types of solid cancer. NF- $\mathrm{BB}$ p65 is the key subunit in this family of transcription factors (16). Typically, $\mathrm{NF}-\kappa \mathrm{B}$ is bound to $\mathrm{I} \kappa \mathrm{B} \alpha$ and exists in the cytosol (17). Due to over-activation of the PI3K/AKT signaling pathway, NF- $\kappa \mathrm{B}$ p65 translocates into the nucleus and subsequently initiates transcription of numerous genes, including MGMT (18).
Excessive activation of $\mathrm{NF}-\kappa \mathrm{B}$ has been previously reported in glioblastoma, and NF- $\mathrm{NB}$ activity has been observed to be higher in tumor tissues compared with normal brain tissues (19). These observations support the hypothesis that combination treatment with TMZ and inhibitors of Akt signaling pathway may achieve promising clinical benefit.

The present study focused on the possible mechanism of BKM120 (a potent selective PI3K inhibitor) in sensitizing C6 glioma cells to TMZ. The findings revealed that BKM120 induced significant downregulation of the PI3K/AKT signaling pathway in C6 glioma. However, the BKM120 monotherapy required a high concentration in order to inhibit viability of $\mathrm{C6}$ cells. Combination treatment with BKM120 and TMZ resulted in a strong synergistic cytotoxicity effect on C6 glioma cells. A low concentration of BKM120 was able to sensitize glioma cells to $\mathrm{TMZ}$. The $\mathrm{IC}_{50}$ of $\mathrm{TMZ}$ significantly decreased from $1,137.67 \pm 271.53$ to $346.67 \pm 134.53 \mu \mathrm{M}$, and the CDIs were close to 0.7 in various TMZ concentrations, which indicated that the TMZ and BKM120 combination treatment had a strong synergistic effect on C6 glioma cells.

Apoptotic defects are present in numerous types of solid cancer, including glioblastoma (20). A previous study demonstrated that apoptosis serves a critical role in TMZ-induced cell death (21). The present study performed Hoechst 33342 and TUNEL staining to investigate morphological changes and rates of apoptosis in various treatment groups. There was a higher level of apoptosis in cells treated with BKM120 and TMZ compared with cells treated with TMZ alone. In addition, principal apoptosis proteins, including caspase enzymes 
and Bax family, were determined. The present study evaluated the level of cleaved caspase-3 expression, which is the most frequently activated protein in the apoptosis process, as well as the expression of Bax in glioma cells treated with TMZ and/or BKM120. Results from western blotting revealed that TMZ and/or BKM120 treatment increased the expression of cleaved caspase- 3 and Bax, and this change was more prominent in the combination treatment group.

The present study investigated MGMT and NF- $\kappa \mathrm{B}$ p65 expression in different treatment groups, with the aim of investigating the association between the PI3K/Akt/NF- $\kappa \mathrm{B}$ signaling pathway and MGMT transcription. Marked increases in MGMT transcription were identified in the TMZ monotherapy group, which was in agreement with a previous study (18). Secondly, no significant change was revealed in the BKM120 monotherapy group compared with the vehicle control group. Notably, MGMT transcription levels were suppressed in cells treated with combination treatment compared with cells treated with TMZ monotherapy. This finding suggested that the BKM120-induced inhibition of MGMT transcription may only be stimulated when activation of MGMT transcription is excessive and aberrant, for example when induced by TMZ. The expression of NF- $\mathrm{B}$ p 65 was increased following treatment with TMZ monotherapy. When treated with BKM120 alone, there were no significant changes in the level of $\mathrm{NF}-\kappa \mathrm{B}$ p65 expression. However, the combination treatment induced a significant decrease in $\mathrm{NF}-\kappa \mathrm{B}$ p 65 expression compared with TMZ treatment alone. This finding was similar to the changes observed in the level of MGMT transcription, which suggests that the PI3K/AKT/NF- $\mathrm{BB} / \mathrm{MGMT}$ signaling pathway may be involved in the synergistic effect of combination treatment.

Due to the limitations of the experimental conditions, the present study did not use RNA interference to demonstrate the direct role of $\mathrm{NF}-\kappa \mathrm{B}$ p65 in the BKM120-induced MGMT reduction. In addition, whether other cancer cell death mechanisms, including autophagy, were involved in the progress of combination treatment requires additional investigation. In the present study, the synergistic cytotoxicity of TMZ and BKM120 on C6 glioma cells was confirmed. Compared with TMZ monotherapy, a combination of TMZ and BKM120 decreased the survival of C6 glioma cells. Additionally, cleaved caspase-3 and Bax expression was increased in cells treated with the combination treatment compared with TMZ monotherapy. There were also significantly higher levels of apoptosis. Furthermore, the present study suggested that BKM120 may be able to inhibit TMZ-induced MGMT transcription, which may be associated with downregulation of $\mathrm{NF}-\kappa \mathrm{B}$ p65. In conclusion, combination therapy of BKM120 and TMZ may be a promising strategy for the treatment of glioblastoma. Furthermore, the present study suggested a mechanism of action for the BKM120 and TMZ combination treatment, for the application of PI3K/Akt inhibitors to reverse TMZ-resistance in clinical treatment.

\section{References}

1. Tate MC and Aghi MK: Biology of angiogenesis and invasion in glioma. Neurotherapeutics 6: 447-457, 2009.

2. Grauer OM, Wesseling P and Adema GJ: Immunotherapy of diffuse gliomas: Biological background, current status and future developments. Brain Pathol 19: 674-693, 2009.
3. Erickson LC, Bradley MO, Ducore JM, Ewig RA and Kohn KW: DNA crosslinking and cytotoxicity in normal and transformed human cells treated with antitumor nitrosoureas. Proc Natl Acad Sci USA 77: 467-471, 1980

4. Pegg AE, Dolan ME and Moschel RC: Structure, function, and inhibition of O6-alkylguanine-DNA alkyltransferase. Prog Nucleic Acid Res Mol Biol 51: 167-223, 1995.

5. Chen L, Han L, Shi Z, Zhang K, Liu Y, Zheng Y, Jiang T, Pu P, Jiang C and Kang C: LY294002 enhances cytotoxicity of temozolomide in glioma by down-regulation of the PI3K/Akt pathway. Mol Med Rep 5: 575-579, 2012.

6. Rodon J, Braña I, Siu LL, De Jonge MJ, Homji N, Mills D, Di Tomaso E, Sarr C, Trandafir L, Massacesi C, et al: Phase I dose-escalation and -expansion study of buparlisib (BKM120), an oral pan-Class I PI3K inhibitor, in patients with advanced solid tumors. Invest New Drugs 32: 670-681, 2014.

7. Livak KJ and Schmittgen TD: Analysis of relative gene expression data using real-time quantitative PCR and the 2(-Delta Delta C(T)) method. Methods 25: 402-408, 2001.

8. Furnari FB, Fenton T, Bachoo RM, Mukasa A, Stommel JM, Stegh A, Hahn WC, Ligon KL, Louis DN, Brennan C, et al: Malignant astrocytic glioma: Genetics, biology, and paths to treatment. Genes Dev 21: 2683-2710, 2007.

9. Butowski NA, Sneed PK and Chang SM: Diagnosis and treatment of recurrent high-grade astrocytoma. J Clin Oncol 24: 1273-1280, 2006.

10. Sarkaria JN, Kitange GJ, James CD, Plummer R, Calvert H, Weller M and Wick W: Mechanisms of chemoresistance to alkylating agents in malignant glioma. Clin Cancer Res 14: 2900-2908, 2008.

11. Brandes AA, Franceschi E, Tosoni A, Blatt V, Pession A, Tallini G, Bertorelle R, Bartolini S, Calbucci F, Andreoli A, et al: MGMT promoter methylation status can predict the incidence and outcome of pseudoprogression after concomitant radiochemotherapy in newly diagnosed glioblastoma patients. J Clin Oncol 26: 2192-2197, 2008.

12. Turriziani M, Caporaso P, Bonmassar L, Buccisano F, Amadori S, Venditti A, Cantonetti M, D'Atri S and Bonmassar E: O6-(4-bromothenyl) guanine (PaTrin-2), a novel inhibitor of O6-alkylguanine DNA alkyl-transferase, increases the inhibitory activity of temozolomide against human acute leukaemia cells in vitro. Pharmacol Res 53: 317-323, 2006.

13. Quinn JA, Jiang SX, Reardon DA, Desjardins A, Vredenburgh JJ, Rich JN, Gururangan S, Friedman AH, Bigner DD, Sampson JH, et al: Phase II trial of temozolomide plus o6-benzylguanine in adults with recurrent, temozolomide-resistant malignant glioma. J Clin Oncol 27: 1262-1267, 2009.

14. Chakravarti A, Zhai G, Suzuki Y, Sarkesh S, Black PM, Muzikansky A and Loeffler JS: The prognostic significance of phosphatidylinositol 3-kinase pathway activation in human gliomas. J Clin Oncol 22: 1926-1933, 2004.

15. Misra UK, Deedwania R and Pizzo SV: Activation and cross-talk between Akt, NF-kappaB, and unfolded protein response signaling in 1-LN prostate cancer cells consequent to ligation of cell surface-associated GRP78. J Biol Chem 281: 13694-13707, 2006.

16. Kumar A, Takada Y, Boriek AM and Aggarwal BB: Nuclear factor-kappaB: Its role in health and disease. J Mol Med (Berl) 82: 434-448, 2004

17. Senftleben U, Cao Y, Xiao G, Greten FR, Krähn G, Bonizzi G, Chen Y, Hu Y, Fong A, Sun SC and Karin M: Activation by IKKalpha of a second, evolutionary conserved, NF-kappa B signaling pathway. Science 293: 1495-1499, 2001.

18. Lavon I, Fuchs D, Zrihan D, Efroni G, Zelikovitch B, Fellig Y and Siegal T: Novel mechanism whereby nuclear factor kappaB mediates DNA damage repair through regulation of $\mathrm{O}(6)$-methylguanine-DNA-methyltransferase. Cancer Res 67: 8952-8959, 2007

19. Huang H, Lin H, Zhang $\mathrm{X}$ and Li J: Resveratrol reverses temozolomide resistance by downregulation of MGMT in T98G glioblastoma cells by the NF- $\mathrm{BB}$-dependent pathway. Oncol Rep 27: 2050-2056, 2012.

20. Igney FH and Krammer PH: Death and anti-death: Tumour resistance to apoptosis. Nat Rev Cancer 2: 277-288, 2002.

21. Roos WP, Batista LF, Naumann SC, Wick W, Weller M, Menck CF and Kaina B: Apoptosis in malignant glioma cells triggered by the temozolomide-induced DNA lesion O6-methylguanine. Oncogene 26: 186-197, 2007. 